\section{Deconstructing light}

Nature Commun. 3, 979 (2012)

Light is intrinsically a combination of electric and magnetic fields. But when photons interact with matter, it is usually the electric interactions that play the dominant role, almost totally obscuring any magnetic effects. Tim Taminiau and co-workers, however, have managed to separate the influence of magnetic and electric dipoles in europium ions, and then to probe magnetic light-matter interactions at the nanoscale.

Emission from an electric and a magnetic dipole differ in terms of their shape and polarization. The ions investigated by Taminiau et al. were scattered throughout a dielectric thin film. Interference effects due to scattering at the top and bottom surfaces of the film further enhanced the differences between electric and magnetic optical transitions, making them distinguishable in energy- and momentum-resolved measurements.

The researchers hope that this technique could become an important tool for nanooptics. They show how it can be used to investigate the changes in the electromagnetic environment as different materials are placed on top of the dielectric thin film.

\section{Smooth transition}

Phys. Rev. Lett. 109, 042003 (2012)

While the Large Hadron Collider at CERN pushes the energy frontier for particle collisions up to the scale of several teraelectronvolts, colliders elsewhere in the world are exploiting lower-energy interactions to fill in more details of the present standard model. The BESIII collaboration - using the upgraded Beijing Electron Positron Collider (BEPCII) at the Intitute of High Energy Physics, Beijing, China - now presents the first measurement of a magnetic dipole transition of the $\psi(3686)$ particle, a so-called charmonium (charm-anticharm quark) state, and a useful guide in understanding more about the strong interactions of quarks.

The collaboration pinpoints the radiative transition of the $\psi(3686)$ to a photon and an $\eta_{c}(2 S)$ particle (the first radial excitation of the $\eta_{c}$ state), followed by the decay of the $\eta_{c}(2 S)$ to a certain mix of kaons and pions (which are mesons made up of lighter up, down and strange quarks), using a modified kinematic fit to guard against 'fake' photons in the detector that would skew the analysis. The measured branching fraction for the magnetic dipole transition turns out to be a good match for theoretical calculations.

\section{A matter of choice}

Nature Photon. http://doi.org/h6c (2012)

Light shows its wave or particle nature depending on the choice of measurement. Jian-Shun Tang and colleagues demonstrate that this long-recognized principle of complementarity is, in fact, closely related to quantum superposition.

To explore the principle of complementarity, John Wheeler proposed a delayed-choice experiment. In a MachZehnder interferometer, light passes through a beamsplitter and branches into two beams that are then recombined in a second beamsplitter, before reaching one of two detectors. When the second beamsplitter is present, interference between the two paths is detected, betraying the wave nature of light; if, however, that beamsplitter is not there, light's particle nature is apparent. In the delayed-choice experiment, the second beamsplitter can be either inserted or removed immediately after the photons have passed through the first one. This arrangement excludes the possibility that, by having access to some hidden information, the photon cunningly 'assumes' its wave or particle property beforehand.

Tang et al. used a special second beamsplitter that is in a quantum superposition of being present and absent,

\section{Laser-guided matter}

Phys. Rev. Lett. 109, 030403 (2012)

Particle-wave duality tells us that a stream of matter can behave in a similar way to a beam of light: Giovanni Gattobigio and colleagues now show how crossing the emission from two lasers creates a matter-wave analogue of an optical beamsplitter. This concept could form an integral part of matter-wave interferometers, which are predicted theoretically to be far more sensitive than their optical counterpart.

Gattobigio etal. optically trapped a cloud of rubidium-87 atoms. Some of these atoms drifted slowly along one of the trapping beams, at a speed of about $13 \mathrm{~mm} \mathrm{~s}^{-1}$. After travelling $700 \mu \mathrm{m}$, the atoms met a second laser beam that crossed the first at an angle of $45^{\circ}$. The researchers waited $200 \mathrm{~ms}$ before measuring the position of the atoms. By increasing the power of the second laser, the researchers could switch from a regime where the atoms were split between travelling along both beams to one where $100 \%$ of the atoms moved along the cross beam.

which allowed them to observe single photons in wave-particle superposition states. The fact that light can behave either as a wave or a particle, or as a superposition of both, redefines the concept of wave-particle duality.

IG

\section{Patch work}

Nature Commun. 3, 975 (2012)

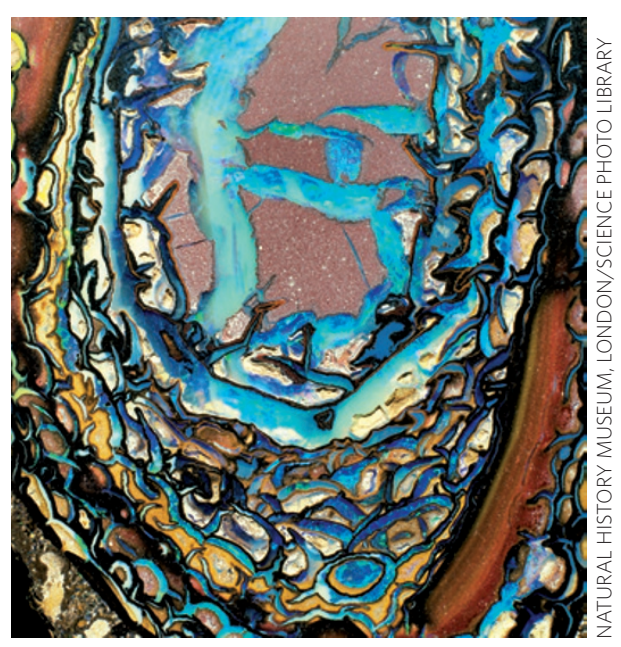

Precious opals owe their striking appearance to a structural periodicity that establishes arrays of interstitial voids, acting as a natural diffraction grating for light. The same periodicity makes self-assembling colloidal structures potentially useful in technologies that rely on enhanced optical transmission. But establishing long-range stability in these synthetic materials has so far proved challenging. Now, Flavio Romano and Francesco Sciortino have come up with a way of stabilizing self-assembled colloidal structures over long length scales.

Particles in these systems effectively interact only with their nearest neighbours, because the potentials act over distances comparable to, or smaller than, the size of the particles themselves. This means that colloidal systems tend to form hybrids comprising crystalline structures that share similar local configurations - such as face-centred-cubic and hexagonal-close-packed lattices.

By engineering colloids patterned with attractive spots on their surface - so-called patchy particles - Romano and Sciortino were able to overcome these difficulties. Their technique, specifically tailored to pattern particles asymmetrically, succeeds in suppressing hexagonal structures, in favour of a pure crystalline state. The strategy is even robust to imperfections in patch placement that may arise from the fabrication process. $A K$

Written by David Gevaux, Iulia Georgescu, Abigail Klopper and Alison Wright 